\title{
Incorporating SPACES recommendations to the COVID-19 ward care approach at the Royal Bournemouth Hospital
}

\author{
Authors: James Tomlinson, ${ }^{A}$ Selina Khan ${ }^{B}$ and Georgina Page ${ }^{C}$
}

The British Thoracic Society have published SPACES (Sharing Patient Assessments Cuts Exposure for Staff) guidance recommending the use of telecommunications as a means of inpatient clinical assessment during the COVID-19 pandemic. The aim of this audit was to reduce face-to-face exposure time during inpatient care of patients with suspected/confirmed COVID-19 using a telecommunications-based approach. 76 patients were included in the audit. 46 patients were included in cycle 1, which measured the average face-to-face time for clinical consultation per patient. 30 patients were included in cycle 2 , whereby history-taking was conducted using telecommunications and, if required, face-to-face physical examination.

Average face-to-face exposure time was reduced to a median of 0 seconds (IQR 0-146.3 seconds) in the telecommunications group, from a median of 312.5 seconds (IQR 178.8-442.3 seconds) in the comparator group $(p<0.0001)$. Patient satisfaction was high with individuals' responses revealing a perception of improved safety by maintaining social distancing. No adverse events were noted.

The audit confirms telecommunications can be integrated successfully into the daily inpatient ward round structure, reducing face-to-face exposure time while maintaining patient satisfaction and safety.

KEYWORDS: Coronavirus, COVID-19, SARS-CoV-2, personal protective equipment, PPE

DOI: $10.7861 /$ clinmed.2020-0613

\section{Introduction}

Coronavirus disease 2019 (COVID-19), caused by severe acute respiratory syndrome coronavirus 2 (SARS-CoV-2), has resulted in a modern-day pandemic affecting over 28 million people, with 916,005 deaths worldwide as of 12 September 2020.' SARS-CoV-2 is transmitted by inhalation or contact with infected droplets

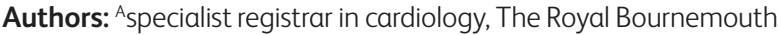
Hospital, Bournemouth, UK; ${ }^{B}$ specialist registrar in ophthalmology, Bristol Eye Hospital, Bristol, UK; ' ${ }^{C}$ consultant in diabetes and endocrinology, The Royal Bournemouth Hospital, Bournemouth, UK with an incubation period thought to range from 2-14 days. ${ }^{2-4}$ Frontline healthcare workers have nearly a 12 -fold increased risk of testing positive for COVID-19 compared to individuals in the general community and workers with inadequate access to personal protective equipment (PPE) are at an even higher risk. ${ }^{5,6}$

Two emergent requirements to maintain our NHS workforce have materialised: to reduce non-essential face-to-face exposure with patients and to preserve the limited supply of PPE for healthcare workers. In response to this, the British Thoracic Society published SPACES (Sharing Patient Assessments Cuts Exposure for Staff), a standardised management approach to ward-based care, recommending the use of telecommunications to perform clinical assessment where possible (see supplementary material, S1).? Implementation of the SPACES approach to ward rounds has the potential to keep staff safe during a time of worldwide shortage in PPE, while also reducing the likelihood of transmission of SARS-CoV-2 to other non-COVID-19 patients. ${ }^{8-12}$

The aim of the audit was to reduce face-to-face exposure time with COVID-19 positive or suspected cases by introducing the use of telecommunications for routine ward round assessment. We retrospectively reviewed the notes to determine any adverse outcomes defined by a failed discharge within 7 days. A subsidiary aim was to both quantify and qualify overall patient satisfaction.

\section{Methods}

Inpatients included in the audit had a confirmed or suspected diagnosis of COVID-19 infection as defined by a fever $>37.7^{\circ} \mathrm{C}$ and one or more of a new persistent cough, coryzal symptoms and/or anosmia.

All patients prior to inclusion had been clerked by a junior doctor and seen by a medical consultant on the post-take ward round and given a working diagnosis. Only follow-up patients with suspected or confirmed COVID-19 undergoing routine ward round review formed the audit cohort.

Patients in cycle 1 (comparison group) were assessed through traditional face-to-face clinical consultation either by a consultant or registrar. Patients in cycle 2 (post-intervention group) had their clinical history taken by telecommunications using either their own mobile phone or personal bedside handset.

Patients receiving teleconsultations gave verbal, informed consent to participate in this audit. Provision of a history template (supplementary material, S2) allowed patients to prepare for the teleconsultation. We recorded the duration 
of the consultation, whether the patient was examined and reason(s) for this (supplementary material, S3). Patient co-morbidities, clinical history and outcomes were also recorded. Following teleconsultation, patients were provided with a satisfaction survey to complete which used a Likert scale (supplementary material, S4). Exclusion criteria included unstable patients with a National Early Warning Score (NEWS) of $>5$, patients with confusion, significant hearing impairment and/or those unable to consent.

The data was analysed using GraphPad Prism 5 software. Non-parametric statistical analysis was performed, including Mann-Whitney $U$ test, median and Interquartile range (IQR). This audit was registered and discussed with the Clinical Governance and Audit Department and had the approval of the clinical director of medicine at the Royal Bournemouth Hospital.

\section{Results}

In total 76 patients were involved in the audit project: 46 patients in cycle 1 and 30 patients in cycle 2. Of this audit group, 9 patients were included in both cycle 1 and cycle 2 . Face-to-face exposure time (less than 2 metres) was reduced to a median of 0 seconds (IQR 0-146.3 seconds) in the telecommunications group from a median of 312.5 seconds (IQR 178.8-442.3 seconds) in the conventional ward round group (Fig 1). 22 of the 30 patients receiving telecommunications did not require physical examination; thus our results demonstrate face-to-face exposure times of 0 seconds on both the 25 th and 50th IQR percentiles. 25 out of the 46 patients in cycle 1 underwent physical examination.

A statistically significant reduction in average face-to-face time was found $(p<0.0001)$. Comparison of total consultation time (history $+/$ - examination) between the telecommunications group (cycle 2) and baseline group (cycle 1), 356.0 seconds and 312.5 seconds respectively, showed an increase in average consultation time in the former that was of marginal significance $(p<0.0505)$. No adverse events were noted.

Patients satisfaction surveys were completed for 20 cases in the telecommunications group. High levels of satisfaction were identified in all but one case, as demonstrated by both median Likert scores and individual responses. 17 of 20 patients felt safer (agree/strongly agree) with clinicians abiding by the 2-metre social distancing government policy, while two were undecided (neither agree nor disagree) and one disagreed. 19 patients felt their expectations had been met, one patient neither agreed nor disagreed. All patients felt satisfied with the care they received (agree/strongly agree). Median Likert scores for all three questions were as follows: 4, 5, and 5 (agree/strongly agree) for questions 1 , 2 and 3 respectively.

Qualitative feedback from patients allowed for further insight into the patient experience. One patient stated, 'I think it was quite important to do the consult via telephone rather than in person in the room with me.' Another patient stated, 'Brilliant idea with mobile phone questionnaire...followed by personal visit - excellent!' Multiple further comments commended the service provided via telecommunications. There was one comment which highlighted the impact of changes to hospital practice due to COVID-19: '... masks etc necessary but changes any conversation, isolation and no visitors also changes the experience, but nonetheless everyone has been very open and friendly to make up for all this...'

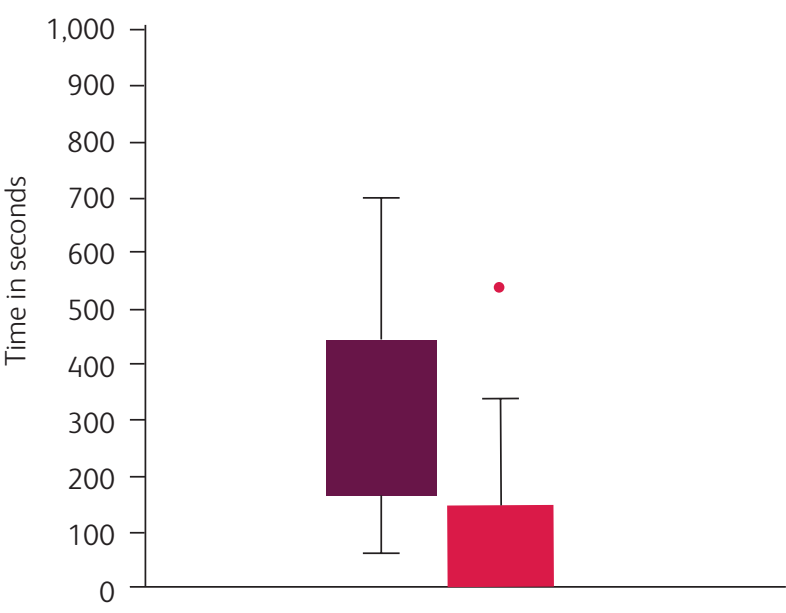

Conventional ward rounds group

Telecommunications group

Fig 1. Face-to-face exposure time with $<2$ metres distancing in the telecommunications group compared to the conventional ward rounds group.

\section{Discussion}

This audit is a novel exploration into the use of telecommunications for inpatients with suspected or confirmed COVID-19. The results show a significant reduction in face-to-face exposure time in patients receiving teleconsultations. This was associated with positive patient feedback and high satisfaction levels, with several patients identifying the importance of telecommunications. Specifically, these individuals' responses highlight their perception of improved safety by ensuring the workforce abide by social distancing government advice.

The average total consultation time in cycle 2 (history +/examination when performed) was longer despite a reduced face-to-face exposure time. This may reflect the impact of a safe environment using telecommunication that allowed for comprehensive, unrushed history-taking aligned with SPACES recommendations (supplementary material, S1). Conversely, in cycle 1 the overall consultation time may have been reduced due to clinician experience and perceptions of medical professionals regarding the need to reduce face-to-face exposure time, thereby minimising the probability of contracting SARS-CoV-2.13,14 Caldwell et al demonstrated an average consultation time of 10 minutes to maintain quality and patient safety in a study of 90 patients seen on routine ward rounds..$^{15}$ The longer consultation times recorded in the telecommunications group are more likely to meet these standards; however, this result was of marginal significance. Average consultation times of 356.0 and 312.5 seconds in the telecommunications and baseline group respectively both fall short of Caldwell's recommendation and Royal College of Physicians guidance recommending 8-12 minutes per patient for a routine ward round of $20-30$ patients. $^{16}$

The majority of patients in both ward round approaches were not physically examined. This is consistent with previously published literature which suggests definitive diagnosis can be reached in most patients by clinical history alone. In a study of 80 patients, definitive 
diagnosis was achieved in 66 out of 80 patients by clinical history alone, with clinical examination being useful in only seven patients and laboratory investigations in a further $7 .^{17}$ This highlights the importance of a thorough clinical history and review of patients' blood tests, imaging and up-to-date vital observations, which are collectively a robust indicator of clinical status. Inpatients that are clinically stable would form the ideal population for a telecoms-only consultation approach. Fewer face-to-face consultations would help reduce the likelihood of direct viral transmission and unnecessary use of PPE. Alongside this, the continued use of monitoring using the NEWS hospital scoring system would indicate earlier on a ward round whether there is any acute deterioration as is currently the standard practice.

The main limitation of this audit is the absence of blinding due to the nature of audit methodology. Therefore, performance bias could have been introduced since clinicians were aware that they were being timed. A modest sample size may have failed to identify a significant increase in total consultation time in cycle 2 Despite this, the cohort in each cycle was large enough to detect a significant and clinically relevant reduction in face-to-face exposure time.

In day-to-day clinical practice, telecommunications could be integrated into the traditional ward round, whereby a history is taken using telecommunications followed by a focused clinical examination if required. This would be safer and more comprehensive than the traditional ward round approach, considering our audit reported only $55 \%$ of patients in the baseline group were examined. An alternative approach would be to vary between teleconsultations only and the traditional ward round approach on alternate days, ie use of telecommunications for mid-week review.

Clinicians are increasingly utilising telecommunications in clinical practice. A systematic review of 57 studies on the use and application of e-consultations in primary care demonstrated improvements in aspects of care delivery, with patients reporting satisfaction with services and improved self-care, communication and engagement with clinicians. ${ }^{18}$ In response to the COVID-19 pandemic, NHS digital and NHSX have effectively transitioned most secondary care outpatient services to telecommunication consultations. General practice has similarly had to adapt to a rapid change of clinical practice and scale up of technology to facilitate the use of teleconsultations. Although the efficacy and safety of video consultations in acute epidemic situations is not yet known, teleconsultations appear to be broadly safe for low-risk patients in the outpatient setting. ${ }^{19}$ The main challenge is applying telecommunications successfully and safely to inpatient care, which was successful so far in our preliminary audit.

By keeping the approach simple and convenient, using patients' mobile phones and bedside handsets, the approach was easily implemented and transferable to other acute inpatient wards, including the acute medical unit. Nursing staff were made aware of our telecommunication approach prior to commencement of the ward round and would inform patients during the routine morning observations. Bedside hygiene was maintained using Clinell ${ }^{\circledR}$ universal wipes to clean the handsets. No additional software or procurement contracts were required to use the technology available.

Implementing the use of telecommunications was not associated with any adverse events although the selection of a small cohort of largely stable patients may have minimised the likelihood of adverse events occurring.

\section{Conclusion}

This audit shows telecommunications can be integrated successfully into the inpatient setting to limit face-to-face exposure and minimise the risk of viral transmission. Our paradigm for teleconsultations relies on careful inpatient selection to optimise patient engagement and maintain patient safety. In the future, we would look at establishing this practice alongside traditional face-to-face ward rounds on alternate days. There is scope to continue this initiative after the COVID-19 pandemic, especially for patients with contractable conditions such as $C$. difficile or if there is a resurgence of SARS-CoV-2 infections.

\section{Supplementary material}

Additional supplementary material may be found in the online version of this article at www.rcpjournals.org/clinmedicine:

S1 - SPACES ward poster

S2 - History template

S3 - Data collection form

S4 - Patient satisfaction form

\section{Acknowledgements}

We would like to acknowledge the following individuals for encouraging and facilitating the completion of this project: Dr Michael Wheble, Dr Augustin Brooks, Dr Girish Babu, Dr Divya Tiwari, Dr David Sell, Dr Earl Williams, Dr Dan Jacyna and the Clinical Governance Department at the Royal Bournemouth Hospital.

\section{References}

1 European Centre for Disease Prevention and Control. COVID-19 situation update worldwide. ECDC, 2020. www.ecdc.europa.eu/ en/geographical-distribution-2019-ncov-cases [Accessed 12 Sept 2020].

2 Yi Y, Lagniton P, Ye S et al. COVID-19: what has been learned and to be learned about the novel coronavirus disease. Int J Biol SCi 2020;16:1753-66.

3 Li Q, Guan X, Wu P et al. Early transmission dynamics in Wuhan, China, of novel coronavirus-infected pneumonia. N Engl J Med 2020;382:1199-1207.

4 Cascella M, Rajnik M, Cuomo A et al. Features, evaluation and treatment Coronavirus (COVID-19). Treasure Island (FL): StatPearls Publishing, 2020. www.ncbi.nlm.nih.gov/books/NBK554776/ [Accessed 23 April 2020].

5 Lai X, Wang M, Qin C et al. Coronavirus Disease 2019 (COVID2019) infection among health care workers and implications for prevention measures in a tertiary hospital in Wuhan, China. JAMA Network Open 2020;3:e209666.

6 Ran L, Chen X, Wang Y et al. Risk factors of healthcare workers with corona virus disease 2019: a retrospective cohort study in a designated hospital of Wuhan in China. Clin Infect Dis 2020, in press (doi: 10.1093/cid/ciaa287).

7 British Thoracic Society. SPACES (Sharing Patient Assessments Cuts Exposure for Staff). BTS, 2020. www.brit-thoracic.org.uk/about-us/ covid-19-information-for-the-respiratory-community/ [Accessed 15 May 2020].

8 Ranney ML, Griffeth V, Jha AK. Critical supply shortages - the need for ventilators and personal protective equipment during the Covid-19 pandemic. N Engl J Med 2020;382:e41.

9 Cook TM. Personal protective equipment during the coronavirus disease (COVID) 2019 pandemic - a narrative review. Anaesthesia 2020;75:920-7. 
10 Livingston E, Desai A, Berkwits M. Sourcing personal protective equipment during the COVID-19 pandemic. JAMA 2020;323: 1912-4.

11 Public Health England. Considerations for acute personal protective equipment (PPE) shortages. PHE, 2020. www.gov.uk/government/ publications/wuhan-novel-coronavirus-infection-prevention-andcontrol/managing-shortages-in-personal-protective-equipment-ppe [Accessed 15 May 2020].

12 Royal College of Physicians. Statement on PHE advice on managing PPE shortages. RCP, 2020. www.rcplondon.ac.uk/news/statementphe-advice-managing-ppe-shortages [Accessed 14 May 2020]

13 Gill D, Gaunt R, Hamdulay S. No substitute for experience: do consultants that have been practising for longer lead faster post-take medical ward rounds? Acute Med 2013;12:141-5.

14 Gawande A. Keeping the coronavirus from infecting health-care workers. New Yorker, 21 March 2020. www.newyorker.com/news/newsdesk/keeping-the-coronavirus-from-infecting-health-care-workers.

15 Herring R, Desai T, Caldwell G. Quality and safety at the point of care: how long should a ward round take? Clin Med 2011:11:20-2.
16 Royal College of Physicians. Consultant physicians working with patients - geriatric medicine. RCP, 2013.

17 Hampton JR, Harrison MJ, Mitchell JR et al. Relative contributions of history-taking, physical examination, and laboratory investigation to diagnosis and management of medical outpatients. BMJ 1975; 2:486.

18 Mold F, Hendy J, Lai YL, de Lusignan S. Electronic consultation in primary care between providers and patients: systematic review. JMIR Med Inform 2019;7:e13042.

19 Greenhalagh T, Wherton J, Shaw S, Morrison C. Video consultations for COVID-19 - an opportunity in a crisis? BMJ 2020;368:m998.

Address for correspondence: Dr James Tomlinson, The Royal Bournemouth Hospital, Castle Lane East, Bournemouth, Dorset BH7 7DW, UK.

Email: jamiesteventomlinson@doctors.org.uk 